\title{
Towards Adaptive, Self-Configuring Networked Unmanned Aerial Vehicles
}

\author{
Nirupama Bulusu ${ }^{*}$ \\ nbulusu@pdx.edu \\ Portland State University \\ Portland, Oregon, USA
}

\author{
Ehsan Aryafar \\ earyafar@pdx.edu \\ Portland State University \\ Portland, Oregon, USA
}

\author{
Feng Liu \\ fliu@pdx.edu \\ Portland State University \\ Portland, Oregon, USA
}

\begin{abstract}
Networked drones have the potential to transform various applications domains; yet their adoption particularly in indoor and forest environments has been stymied by the lack of accurate maps and autonomous navigation abilities in the absence of GPS, the lack of highly reliable, energy-efficient wireless communications, and the challenges of visually inferring and understanding an environment with resource-limited individual drones. We advocate a novel vision for the research community in the development of distributed, localized algorithms that enable the networked drones to dynamically coordinate to perform adaptive beam forming to achieve high capacity directional aerial communications, and collaborative machine learning to simultaneously localize, map and visually infer the challenging environment, even when individual drones are resource-limited in terms of computation and communication due to payload restrictions.
\end{abstract}

\section{CCS CONCEPTS}

- Networks $\rightarrow$ Location based services; Cross-layer protocols; - Computing methodologies $\rightarrow$ Ensemble methods.

\section{KEYWORDS}

drones, localization, adaptive beam forming, collaborative visual inference, federated learning

\section{ACM Reference Format:}

Nirupama Bulusu, Ehsan Aryafar, and Feng Liu. 2021. Towards Adaptive, Self-Configuring Networked Unmanned Aerial Vehicles. In The 7th Workshop on Micro Aerial Vehicle Networks, Systems, and Applications (DroNet) (Dronet '21), June 24, 2021, Virtual, WI, USA. ACM, New York, NY, USA, 6 pages. https://doi.org/10.1145/3469259.3470488

\section{INTRODUCTION}

Unmanned aerial vehicles (UAVs), or drones, are envisioned to be the ultimate disruptive technology for industries spanning infrastructure, agriculture, transport, entertainment, security and mining. PricewaterhouseCoopers forecasts the annual global market for drone powered business solutions to be at $\$ 127$ billion. Despite

\footnotetext{
*All authors contributed equally to this research.

Permission to make digital or hard copies of all or part of this work for personal or classroom use is granted without fee provided that copies are not made or distributed for profit or commercial advantage and that copies bear this notice and the full citation on the first page. Copyrights for components of this work owned by others than ACM must be honored. Abstracting with credit is permitted. To copy otherwise, or republish, to post on servers or to redistribute to lists, requires prior specific permission and/or a fee. Request permissions from permissions@acm.org.

Dronet '21, fune 24, 2021, Virtual, WI, USA

(C) 2021 Association for Computing Machinery.

ACM ISBN 978-1-4503-8599-2/21/06...\$15.00

https://doi.org/10.1145/3469259.3470488
}

burgeoning research and commercial activity on individual drones, particularly outdoors, their full technology potential is yet to be realized.

The vision of this paper is to amplify these capabilities and application opportunities by effectively harnessing networks of drones operating in concert to overcome failures or resource limitations of individual drones. The ensemble of drones has vastly superior navigation, inference, communication and task capabilities than any single resource limited drone could possess. To enable this vision, we must investigate scalable coordination mechanisms for networks of drones, with the ensemble as a whole being more robust, versatile and powerful than individual drones.

The challenges faced in designing networks of drones are best explicated by the following scenarios. Structural faults in extremely large buildings (e.g. fatigue cracks) require frequent visual inspection by engineers, which is time consuming and possibly unreliable. Static sensors like strain gauges or accelerometers may be difficult to deploy and maintain in some locations (e.g. calibrate or replace batteries). Also the strain profile around cracks is complex, which presents challenges for quantifying the crack tip location. Moreover, a crack often deviates from projected trajectory making it difficult to select appropriate sensor locations. It would be extremely cumbersome for a single micro-aerial vehicle to inspect a vast area or relay or even store all the visual information. Networked UAVs can inspect complex, difficult to access areas in outdoor/indoor environments for fatigue cracks.

A second scenario concerns forest fires. For firefighters, as seen during the 2020 West Coast wildfires, combating forest fires and determining fire spread is extremely challenging, particularly under windy conditions. Networked UAVs can map the forest contours and map areas not safe for humans, or provide data for mobile augmented reality mapping of the environment. Multi-hop wireless networks could enable communication and coordination among autonomous drones, in both planned and unplanned environments and configurations. However, dynamic channel conditions and drones' mobility pose significant challenges to reliable high capacity aerial networking. These challenges are exacerbated by power, sensing, and computation limitations on each drone, which further complicate the interactions between high level behavior and lower level communication characteristics.

In our research project, we are investigating, and call upon the research community to also develop, test and characterize instruments that provide scalable, application-driven services including mapping and collaborative visual inference using a diverse collection of communicating mobile autonomous aerial vehicles, even in challenging environments such as indoors, where GPS is not available. We emphasize that the drones are not merely communicating and forwarding data but are actively coordinating to accomplish 
various tasks. These nodes will be autonomous in that their movements will not be human controlled. They coordinate with each other to morph into a wide variety of network formations and configurations. These capabilities could be enabled by the research and development of distributed algorithms for robust navigation, collaborative vision, and aerial communications. Note that mobile drones, for aspects of their development will require communication to a backend machine learning (ML) supercomputer. We emphasize that most of these drones possess modest sensing, computational and communication resources. The collection as a whole is an example of an adaptive, self-configuring networked UAV instrument.

The networked drone instrument development requires a crossdisciplinary approach using ideas from computer vision, machine learning, wireless communication and networking, FPGA programming, and circuit design. The key hardware challenges include: (i) design of a fully programmable multi-antenna platform that can provide maximal three-dimensional connectivity (i.e., communication to/from the top/bottom/sides with minimal blockages from the UAV body); (ii) appropriate sensory equipment and PCB to increase contextual awareness of the UAV (e.g., speed, direction, elevation); and (iii) design of appropriate circuitry (power, camera feed) to mount a high performance GPU on the drone. The key software challenge would be to develop both user and network drone interfaces to fully customize the operation of each and every drone.

Development of such instruments will enable applications such as structural health monitoring while providing several significant new capabilities not available in any instrument currently available from a vendor. These include:

- Robust Aerial Navigation Without GPS: Existing UAVs rely on GPS receivers for positioning. With appropriate hardware (e.g., sensory PCBs) and software tools for accurate GPS-less aerial navigation.

- Collaborative Visual Inference: Existing UAVs rely on onboard processing, when available, to identify and categorize objects. The increased processing power drains the battery and reduces flight time. Networked drone instruments pool processing power of multiple drones for collaborative visual inference with much reduced power and increased flight time.

- Aerial Wireless Communications: Existing UAVs rely on offthe-shelf wireless cards with omni antennas for communication. These cards implement existing wireless technologies with no/limited programmability. Fully programmable multiantenna aerial platform that could be used for development of new directional aerial communication protocols and networks.

\section{ROBUST AERIAL NAVIGATION SANS GPS}

Aerial navigation is crucial to realize autonomous operation and applications of drones. In the outdoor arena, drones navigate using Global Positioning System (GPS) receivers. Companies such as AirMap [3] provide accurate, reliable and trustworthy low-altitude navigational data and communication tools to the drone industry. GPS and navigational data are not available in certain environments, such as indoors and dense forest trails prevalent in the Pacific Northwest.

Besides prior work on GPS-denied drone navigation[10], state-ofthe-art techniques such as [23] rely on weight-contributing sensors such as LIDAR intended for heavier drones. Vision-based drones could potentially address perception and control in challenging environments. ${ }^{1}$ We propose to use cameras on drones to understand and navigate the environment, with the local GPU enabling onboard AI. This approach adapts and greatly extends early work on visual mapping (to enable an individual drone to navigate forest trails without GPS, TrailNet [41]) to networked coordinating UAVs.

Why the need for a visual mapping approach? A multitude of sensors (e.g. radio, acoustic, IMU, cameras, laser and LIDAR), can be potentially deployed on drones to aid navigation. Because drone cameras are inherently vital for many applications, their dual use for navigation overcomes the reliance on other weight-contributing sensors (eg. LIDAR). WiFi mapping based mobile navigation methods[44] have inherent accuracy limits. Technologies such as WiTrack[1] that track human subjects using multi-antenna base stations are promising, but require a network of previously deployed base stations that may not be feasible. Our approach also sidesteps the need for planning advocated by recent work[17].

A short term research thrust is to develop an individual drone's camera-based navigation system using deep neural networks (DNNs) to enable autonomously navigation in the absence of GPS (indoors, forest trails). The long term goal is to enable a network of drones to asynchronously coordinate to navigate their environment.

(1) Individual Drone Navigation. We are exploring solutions for the problem of individual drone navigation that combine (i) estimating lateral offset and view orientation with convolutional neural networks (CNNs) (ii) object detection with $\mathrm{CNN}$ and (iii) obstacle detection and avoidance with monocular SLAM, rather than stereo-based matching to minimize system complexity. For any path of interest (e.g. indoor corridors, forest trails), it is important to estimate both the three orientations (facing left, center, facing right) and the three lateral offsets (shifted left, centered, shifted right) with respect to the path center to avoid drones crashing into obstacles due to measurement errors. Because training DNNs requires a lot of data and computation, advanced infrastructure (e.g. an NVIDIA DGX Workstation) would comprise a vital part of such infrastructure. For obstacle detection and avoidance, we plan to extend the direct sparse odometry (DSO) method[16], which can compute semi-dense 3D maps of the environment and is computationally efficient. Investigation of methods to make DNNs robust to potential fooling attacks as proposed in [35] would be required.

(2) Evaluation. We plan to test our system by autonomously navigating in several indoor environments, including (i) a straight corridor (ii) a corridor with several turns, and (iii) a staircase. To extend the training network for indoor environments, we plan to use a three-camera rig in which 3 GoPro cameras (aimed left 30 degrees, straight, and right 30 degrees) are mounted on a $1 \mathrm{~m}$ bar. We have such a rig currently in our laboratory. Our evaluation criteria are autonomy, measured

\footnotetext{
${ }^{1}$ https://www.seas.upenn.edu/ loiannog/workshopIROS2018uav/index.html
} 
by the percentage of time that the UAV does not need to be overridden by a human operator to prevent collision with other objects.

(3) Analysis. The key trade-offs are associated with navigation autonomy as a function of flying time. For example, the accuracy can be optimized with the addition of a LIDAR laser range finder ${ }^{2}$ to improve the distance-to-ground estimation, but the additional weight might limit the drone's total flying time. Our goal should be to identify the optimal sensing capabilities that maximize autonomy without sacrificing flying time.

(4) Augmenting the Solution with a Federated Coordinate System. Using iterative multi-lateration algorithms originally developed for wireless sensor networks [8], drones can compute ranges to their neighbors (from various ranging methods, including radio as a baseline) and use these relative distances to form a globally rigid and unique coordinate system. However, current methods used in practice are not guaranteed to find the correct localization, even when it is uniquely determined by input distances. To address this problem, we are developing new algorithms that leverage sensor perturbation [50], with a study of techniques for improving the algorithm's robustness to measurement noise, and strategies for reducing the total number of measurements needed by the algorithm. The key idea in our approach is for our $\mathrm{Si}$ multaneous Localization and Mapping (SLAM) algorithms to use waypoints (geospatial markers) for objects that were previously classified by other drones in the network to maintain spatial relationships between drones. Stereo DSO can be enabled by having drones working in pairs instead of the monocular SLAM used in individual drone navigation to make solutions more robust in the absence of photometric calibration[45]. A fruitful area of study is the impact on navigation autonomy from the federation of data from different drones.

\section{COLLABORATIVE VISUAL INFERENCE}

A common functionality across applications is for drones to detect and classify subjects, objects and phenomena of interest. These problems are not unique to UAVs. Self-driving cars have driven the development of machine learning algorithms to detect and classify a variety of objects and subjects, such as pedestrians, road signs and other cars [42]. However, drones bring in unique challenges because their payload restrictions severely and simultaneously limit both on-board compute and communication.

It is both computational challenging and energy infeasible for an individual drone to perform in-situ comprehensive classification of complex scenarios. Nor can it offload all raw data to the cloud! Merely adapting machine learning approaches from other domains will not suffice and new approaches must be explored. Note that our proposed work exploits recent advances in machine learning that are supplanting traditional approaches to computer vision. It is thus complementary to prior literature on cooperative control of drones, such as [12, 45], cooperative surveillance and pursuit [27], edge

${ }^{2}$ Lidar-Lite v3 optical distance measurement sensor, http://buy.garmin.com/enUS/US/p/557294 computing based solutions for processing visual information[13, 22] and task assignment and flocking algorithms [10].

Our plan is to investigate collaborative visual inference to enable low-latency visual analysis on resource-limited networked drones. Specifically, while the computer vision capability of each drone is limited, multiple networked drones can collectively perform highly accurate visual inference for complicated scenes. In addition, we will explore federated learning to collaboratively and continuously improve the computer vision models deployed in drones. ${ }^{3}$ It enables edge devices, particularly mobile phones, to collaboratively learn a shared classification model. Each edge device updates its current machine learning model by learning from data it collects, and then summarizes the changes as a small focused update. Only this update to the model is sent to the server in the cloud, where it is immediately aggregated with other devices' updates to improve the shared model [2]. Such a strategy helps protect user privacy while continuously improving the performance of machine learning models on resource-limited edge devices. This approach has never previously been attempted for visual inference on drones.

Three research problems must be solved to enable collaborative visual inference on drones.

Collaborative visual inference. We plan to explore ensemble learning to aggregate the outputs from networked drones to enable highly accurate visual inference. Ensemble learning is a very effective machine learning technique that can aggregate multiple weak classifiers into a powerful one [36]. Existing ensemble learning algorithms jointly train all the weak classifiers to optimize the final performance on a powerful server. This is feasible for networked drones only before they are deployed. Our idea is to aggregate the outputs from individual drones that cover an overlapped area in the scene. As shown in our previous work [32], an intelligent aggregation mechanism can be developed to effectively ensemble multiple outputs such that the final result outperforms each individual one. Different from the existing work where individual models take exactly the same input, the input to each drone is different from each other. Neighboring drones capture different views of the same scene. This imposes challenges for ensembling, but also provides an important advantage in that multiple views of the same scene can be used for better visual analysis. We plan to build upon our existing ensembling work to develop dedicated ensembling algorithms for networked drones.

Continuous model improvement. We will investigate how to leverage the processors on drones to compute higher quality model updates for federated learning than a few simple steps in a stochastic gradient descent [11]. We will also study methods to aggregate model updates in a scalable, efficient and fault-tolerant way. To conserve energy and significantly reduce model upload communication costs, potentially by a factor of up to $100 x$, we will develop compressed update protocols by combining existing approaches such as random rotations and quantization and developing new ones as well. We will evaluate these protocols using extensive simulations, with the objective of identifying optimal model compression methods and ratios that minimize bandwidth and energy consumption without diminishing accuracy. As part of our analysis, we will (i) evaluate object detection accuracy as a function of model update

\footnotetext{
${ }^{3}$ http://research.googleblog.com/2017/04/federated-learning-collaborative.html
} 
frequency and compression ratios, (ii) measure and characterize the energy savings enabled by the compressed update protocols, and finally, (iii) develop adaptive fidelity algorithms that can dynamically configure the compression parameters in response to desired detection accuracy. The best methods could be implemented in the collaborative visual inference framework.

Light-weight model development. A compact and fast computer vision model is not only critical for deployment in drones, but also important to minimize the communication cost when transmitting the updated model from the server to the drones. The state-of-the-art computer vision algorithms mostly are built upon deep neural networks. While deep neural network models are typically deployed in data centers, light-weight neural network architectures are emerging to support real-time computer vision on resource-limited edge devices, such as ShuffleNet [48] and MobileNet [24]. There are also other technologies to develop lightweight network models, such as pruning [31] and using low-bit models [14]. However, employing such technologies still requires a significant amount of trial-and-error efforts from deep learning experts. We plan to explore the emerging neural network architecture searching algorithms to automatically find a low-bit deep network for each target application that meets the resource limits from drones while still achieving high visual inference performance [15].

\section{MOBILITY MANAGEMENT WITH DIRECTIONAL AERIAL COMMUNICATION}

Distributed implementation of machine learning algorithms across a group of drones requires high capacity communication links between the drones. A natural solution to increase the capacity is to employ multiple antennas at each drone. The plurality of antennas can then be used to create directional beam patterns that increase the SNR (e.g., with a switched beamforming architecture as depicted in Fig. 1(a)). We note some recent work and progress on increasing capacity of micro-aerial vehicles using antenna arrays [21, 34, 39] and mmWave wireless mesh backhauls (e.g. see SkyHaul[40]).

We emphasize however, that directional aerial communication reduces the beam width which makes the communication more susceptible to link failures due to mobility. We propose a two-pronged approach to address the problem of mobility management with directional links. First, we address the problem of beam management such as beam identification and beam tracking between two mobile drones, and develop solution with reduced overhead and energy consumption. Next, we look at the mobility of drones as an opportunity to enhance the network performance and study how drones can optimize their relative locations to build a robust and high capacity network. While here we focus on the interplay between mobility and directional communication, we emphasize that the design of networked drone instruments will enable research in many other relevant research areas. For example, it will allow us to realize different radio architectures (such as switched beam, adaptive beam, and full-duplex) and study their relative performance in aerial contexts or to design and test optimal computation splitting algorithms that offload blocks of computation to a ground station for reduced computation latency and energy consumption.

\subsection{Beam Management}

Beam management in multi-antenna systems with switched beamforming is composed of two parts: (i) beam identification: a mechanism to find the appropriate $\mathrm{Tx}$ and $\mathrm{Rx}$ beam patterns between the two ends of the communication, and (ii) beam tracking: an on-going estimation that, starting from the current steering directions, infers how the directions evolve over time. In existing switched beam wireless communication systems, beam identification is managed through beam search (e.g. exhaustive search) [4$6,18,25,26,28-30,33,38,46,49]$. Beam tracking, on the other hand, is managed through transmission of reference signals and estimation of the direction-of-arrival [9, 19, 20, 37, 47]. Both of these approaches add significant overhead to the communication and hence reduce the performance in terms of UAV energy consumption and link throughput. Our goal here is to develop and evaluate mechanisms for beam management with significantly reduced energy and overhead.

(1) Beam Management. We will develop and study compressive sensing solutions for the problem of beam management in aerial networks. Our key idea is to send a small series of beamformed reference signals in only a few directions such that sufficient measurements of the channel are taken. This measurement codebook allows us to sample the channel space and the feedback from the codebook transmission can be used to reconstruct the channel for the purpose of beam management. We will leverage our aerial testbed to study the performance of our solution in aerial channels and across different frequency bands that are supported by our softwaredefined radios.

(2) Overhead Analysis. We will study the tradeoffs associated with compressive sensing channel estimation and beam management. For example, the measurement codebook can be optimized to increase the beamforming gain (by probing across more directions, which increases the overhead as well as the accuracy in identifying the best beams) or coverage (e.g., beam search with wide beams, which reduces the overhead and accuracy). We will characterize these tradeoffs as a function of number of antennas and will leverage the platform to evaluate them in real aerial channels. We will also compare our solution against state-of-the-art beam management techniques in terms of overhead and energy consumption.

(3) Augmenting the Solution with Context-Awareness. We will study how context-awareness (e.g., information about current location, speed, or historical data) can improve the performance of beam management. For example, consider two drones that are in a Line-of-Sight (LoS) channel state with respect to each other. In our recent work [7], we showed that it is possible for drones to infer this information $(\mathrm{LoS} / \mathrm{nLoS}$ channel state) from existing sector sweep or beam management data, without adding any additional overhead. Now if drones are aware of their relative locations, they can simply pick the beams that point to the LoS direction (i.e., each other) without the need to perform beam management. We will update our beam management algorithms from the previous subsections with such context-aware solutions and 


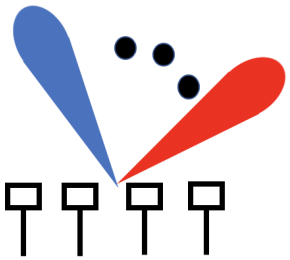

(a)

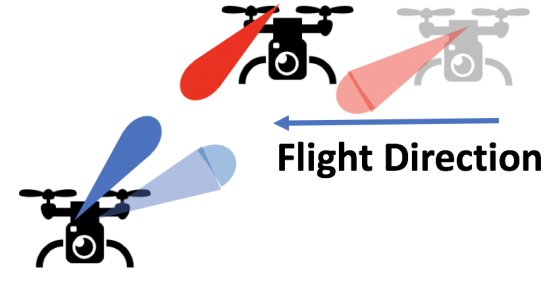

(b)

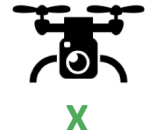

$x$

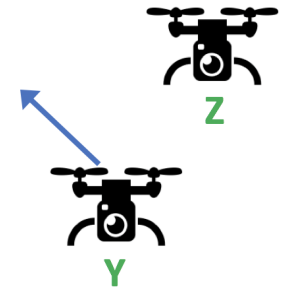

(c)

Figure 1: (a): In a switched beam architecture a set of pre-determined beams are available, (b): beam identification and beam tracking between a pair of drones, and (c): mobile drones optimize their relative locations to enhance network-wide performance. Here, $\mathrm{Y}$ moves to a location between $\mathrm{X}$ and $\mathrm{Z}$ and acts as a relay to increase throughput between $\mathrm{X}$ and $\mathrm{Z}$ (we assume $\mathrm{X}$ and $\mathrm{Z}$ cannot change their locations).

will leverage our aerial testbed to evaluate their real-world performance.

\subsection{Leveraging the UAV Mobility for a Self-Healing Aerial Network}

Our objective is to exploit UAV mobility to create a self-healing aerial network. For example, when UAVs are equipped with directional beams, it may not be possible for each drone to communicate in the entire 360 degree of Azimuth and 180 degree of elevation due to per-element directional antenna patterns [43] or due to the body of the UAV blocking it from transmission in certain directions. As a result, two UAVs may not be able to communicate with each other in a certain relative position, but they may be able to restore the communication if they change their positions. Our overall goal in this section is to develop algorithms that exploit UAV mobility to improve the capacity and robustness of communication.

(1) Mobility Optimization Tradeoffs. We will study the tradeoffs associated with exploiting UAV mobility for the purpose of improving the communication performance. We will first consider a single pair of UAVs and experimentally evaluate the variations in SNR as one UAV moves around the other UAV (in the entire three-dimensional space) in both LoS and nLoS channel conditions. We will also evaluate the impact of beam management solution and the number of antennas on these SNR variations. We will then build a model that characterizes the increase in SNR and the loss in energy expenditure that are associated with exploiting drone mobility for improved link quality.

(2) Algorithms for Optimal Mobility in a Network of Drones. Our goal in this task would be to develop a network wide problem formulation for optimal mobility management that takes into account the model and tradeoffs from the previous subsection as well as the individual mobility and radio capabilities of each drone (e.g., number of antennas on the drone or whether a drone can change its position). Note that two drones independently changing their position may be redundant or even decrease the performance. Thus, we need a distributed mobility optimization framework wherein drones collaboratively change their positions with the objective of maximizing network-wide performance.
(3) Evaluation Across Contexts. Our final task would be to evaluate the performance of our networked drone mobility management algorithms across different aerial contexts (e.g., when drones are deployed to extend cellular capacity or a search and rescue mission) and propagation environments (both indoors and outdoors). We will leverage the proposed networked UAV infrastructure to experimentally evaluate the performance of our algorithms in such contexts and environments.

\section{REFERENCES}

[1] F. Adib, Z. Kabelac, and D. Katabi. 2015. Multi-Person Localization via RF Body Reflections. In 12th USENIX Symposium on Networked Systems Design and Implementation. 279-292.

[2] D. Aiger, B. Allen, and A. Golovinskiy. 2017. Large-Scale 3D Scene Classification With Multi-View Volumetric CNN. ArXiv e-prints (Dec. 2017). arXiv:1712.09216 [cs.CV]

[3] AirMap. 2021. (2021). http://www.airmap.com

[4] A. Alkhateeb, O. E. Ayach, G. Leus, and R. W. Heath. 2014. Channel estimation and hybrid precoding for millimeter wave cellular systems. In IEEE fournal of Selected Topics in Signal Processing.

[5] A. Alkhateeb, G. Leusz, and R. W. Heath. 2015. Compressed sensing based multi-user millimeter wave systems: How many measurements are needed? In Proceedings of IEEE ICASSP.

[6] E. Aryafar and A. K. Haddad. 2015. $F D^{2}$ : a directional full duplex communication system for indoor wireless networks. In Proceedings of IEEE INFOCOM.

[7] E. Aryafar, J. Zhu, S. Singh, M. Akdeniz, W. Lee, and N. Himayat. 2016. Method for LoS-nLoS State Identification in mmWave Sector Sweep. In Submitted to United State Patent Office (USPTO), filing number PCT/US2016/066565.

[8] J. Aspnes, T. Eren, D. K. Goldenberg, A. S. Morse, W. Whiteley, Y. R. Yang, B. D. O. Anderson, and P. N. Belhumeur. 2006. A Theory of Network Localization. IEEE Transactions on Mobile Computing 5, 12 (Dec 2006), 1663-1678.

[9] J. Bae, S. H. Lim, J. H. Yoo, and J. W. Choi. 2017. New beam tracking technique for millimeter wave-band communications. In arXiv:1702.00276.

[10] G. Balamurugan, J. Valarmathi, and V.P. S. Naidu. 2016. Survey on UAV navigation in GPS denied environments. In 2016 International Conference on Signal Processing, Communication, Power and Embedded System (SCOPES). 198-204.

[11] L. Bottou, F. E. Curtis, and J. Nocedal. 2016. Optimization Methods for Large-Scale Machine Learning. ArXiv e-prints (June 2016). arXiv:1606.04838 [stat.ML]

[12] P. R. Chandler, M. Pachter, and S. Rasmussen. 2001. UAV cooperative control. In Proceedings of the 2001 American Control Conference. (Cat. No.01CH37148), Vol. 1. 50-55 vol.1.

[13] Quan Chen, Hai Zhu, Lei Yang, Xiaoqian Chen, Sofie Pollin, and Evgenii Vinogradov. 2021. Edge Computing Assisted Autonomous Flight for UAV: Synergies between Vision and Communications. IEEE Communications Magazine 59, 1 (2021), 28-33. https://doi.org/10.1109/MCOM.001.2000501

[14] Matthieu Courbariaux, Itay Hubara, Daniel Soudry, Ran El-Yaniv, and Yoshua Bengio. 2016. Binarized neural networks: Training deep neural networks with weights and activations constrained to +1 or-1. arXiv preprint arXiv:1602.02830 (2016).

[15] Thomas Elsken, Jan Hendrik Metzen, and Frank Hutter. 2019. Neural Architecture Search: A Survey. Journal of Machine Learning Research 20, 55 (2019), 1-21. 
[16] J. Engel, V. Koltun, and D. Cremers. 2016. Direct Sparse Odometry. CoRR abs/1607.02565 (2016). arXiv:1607.02565

[17] A. Faust, O. Ramirez, M. Fiser, K. Oslund, A. Francis, J. Davidson, and L. Tapia. 2017. PRM-RL: Long-range Robotic Navigation Tasks by Combining Reinforcement Learning and Sampling-based Planning. ArXiv e-prints (Oct. 2017). arXiv:1710.03937 [cs.AI]

[18] B. Gao, Z. Xiao, L. Su, Z. Chen, D. Jin, and L. Zeng. 2015. Multi-device multipath beamforming training for $60-\mathrm{GHz}$ millimeter-wave communications. In Proceedings of IEEE ICC.

[19] X. Gao, L. Dai, Y. Zhang, T. Xie, X. Dai, and Z. Wang. 2017. Fast channel tracking for terahertz beamspace massive MIMO systems. In IEEE Transactions on Vehicular Technology.

[20] N. Garcia, H. Wymeersch, and D. Slock. 2017. Optimal robust precoders for tracking the AoD and AoA of a mm-Wave path. In arXiv:1703.10978.

[21] Jesus Garza, M. Panduro, Alberto Reyna, G. Romero, and C. Rio. 2016. Design of UAVs-Based 3D Antenna Arrays for a Maximum Performance in Terms of Directivity and SLL. International fournal of Antennas and Propagation 2016 (2016), 1-8.

[22] Samira Hayat, Roland Jung, Hermann Hellwagner, Christian Bettstetter, Driton Emini, and Dominik Schnieders. 2021. Edge Computing in 5G for Drone Navigation: What to Offload? IEEE Robotics and Automation Letters 6, 2 (2021), 2571-2578. https://doi.org/10.1109/LRA.2021.3062319

[23] G. Hemann, S. Singh, and M. Kaess. 2016. Long-range GPS-denied aerial inertial navigation with LIDAR localization. In 2016 IEEE/RSF International Conference on Intelligent Robots and Systems. 1659-1666.

[24] A. Howard, M. Sandler, G. Chu, L. Chen, B. Chen, M. Tan, W. Wang, Y. Zhu, R. Pang, V. Vasudevan, et al. 2019. Searching for mobilenetv3. arXiv preprint arXiv:1905.02244 (2019).

[25] S. Hur, T. Kim, D. J. Love, J. V. Krogmeier, T. A. Thomas, and A. Ghosh. 2013. Millimeter wave beamforming for wireless backhaul and access in small cell networks. In IEEE Transactions on Communications.

[26] IEEE 802.11 WG. 2012. IEEE 802.11ad, Amendment 3: Enhancements for Very High Throughput in the $60 \mathrm{GHz}$ Band. (2012).

[27] J.L.Fargeas, P. Kabamba, and A. Girard. 2015. Cooperative Surveillance and Pursuit Using Unmanned Aerial Vehicles and Unattended Ground Sensors. Sensors 15, 1 (2015), 1365-1388.

[28] J. Lee, G. T. Gil, and Y. H. Lee. 2014. Exploiting spatial sparsity for estimating channels of hybrid MIMO systems in millimeter wave communications. In Proceedings of IEEE GLOBECOM.

[29] X. Liu, A. Sheth, M. Kaminsky, K. Papagiannaki, S. Seshan, and P. Steenkiste. 2009. DIRC: increasing indoor wireless capacity using directional antennas. In Proceedings of ACM SIGCOMM.

[30] X. Liu, A. Sheth, M. Kaminsky, K. Papagiannaki, S. Seshan, and P. Steenkiste 2010. Pushing the envelope of indoor wireless spatial reuse using directional access points and clients. In Proceedings of ACM MOBICOM.

[31] Zhuang Liu, Mingjie Sun, Tinghui Zhou, Gao Huang, and Trevor Darrell. 2018. Rethinking the value of network pruning. arXiv preprint arXiv:1810.05270 (2018).

[32] Long Mai, Yuzhen Niu, and Feng Liu. 2013. Saliency aggregation: A data-driven approach. In Proceedings of the IEEE Conference on Computer Vision and Pattern Recognition. 1131-1138.

[33] R. Mendez-Rial, C. Rusu, A. Alkhateeb, N. Gozalez-Prelcic, and R. W. Heath. 2015. Hybrid MIMO architectures for millimeter wave communications: phase shifters or switches?. In IEEE Access.

[34] Mohammad Mozaffari, Walid Saad, Mehdi Bennis, and Mérouane Debbah. 2019 Communications and Control for Wireless Drone-Based Antenna Array. IEEE Transactions on Communications 67, 1 (2019), 820-834. https://doi.org/10.1109/ TCOMM.2018.2871453

[35] A. Nguyen, J. Yosinski, and J. Clune. 2014. Deep Neural Networks are Easily Fooled: High Confidence Predictions for Unrecognizable Images. CoRR abs/1412.1897 (2014). arXiv:1412.1897

[36] David Opitz and Richard Maclin. 1999. Popular ensemble methods: An empirical study. Fournal of artificial intelligence research 11 (1999), 169-198.

[37] J. Palacios, D. D. Donno, and J. Widmer. 2017. Tracking mmWave channel dynamics: fast beam training strategies under mobility. In Proceedings of IEEE INFOCOM.

[38] S. Payami, M. Shariat, M. Ghoraishi, and M. Dianati. 2015. Effective RF codebook design and channel estimation for millimeter wave communication systems. In Proceedings of IEEE ICC.

[39] Alberto Reyna, Jesus Garza, Omar Elizarraras, M. Panduro, Luz I. Balderas, and María de la Luz Prado. 2021. 3D random virtual antenna arrays for FANETs wireless links. Telecommunication Systems (2021), 1-9.

[40] Ramanujan K. Sheshadri, Eugene Chai, Karthikeyan Sundaresan, and Sampath Rangarajan. 2020. SkyHaul: An Autonomous Gigabit Network Fabric in the Sky. CoRR abs/2006.11307 (2020). arXiv:2006.11307 https://arxiv.org/abs/2006.11307

[41] N. Smolyanskiy, A. Kamenev, J. Smith, and S. Birchfield. 2017. Toward Low Flying Autonomous MAV Trail Navigation using Deep Neural Networks for Environmental Awareness. CoRR abs/1705.02550 (2017). arXiv:1705.02550
[42] A. Teichman and S. Thrun. 2011. Practical object recognition in autonomous driving and beyond. In Advanced Robotics and its Social Impacts. 35-38.

[43] E. Teng, J. D. Falcao, and B. Iannucci. 2017. Holes-in-the-sky: a field study on cellular-connected UAS. In Proceedings of IEEE International Conference on Unmanned Aircraft Systems (ICUAS).

[44] H. Tran, S. Pandey, and N. Bulusu. 2017. Poster: Online Map Matching for Passive Indoor Positioning Systems. In Proceedings of the 15th Annual International Conference on Mobile Systems, Applications, and Services (Niagara Falls, New York, USA) (MobiSys '17). ACM, New York, NY, USA, 175-175.

[45] J. Wang, C. Jiang, Z. Han, Y. Ren, R. Maunder, and L. Hanzo. 2017. Taking Drones to the Next Level: Cooperative Distributed Unmanned-Aerial-Vehicular Networks for Small and Mini Drones. IEEE Vehicular Technology Magazine 12 (07 2017), 73-82.

[46] J. Wang, Z. Lan, C. Pyo, T. Baykas, C. S. Sum, M. A. Rahman, J. Gao, R. Funada, F. Kojima, H. Harada, and S. Kato. 2009. Beam codebook based beamforming protocol for multi-Gbps millimeter-wave WPAN systems. In IEEE fournal on Selected Areas in Communications.

[47] C. Zhang, D. Guo, and P. Fan. 2016. Mobile millimeter wave channel acquisition, tracking, and abrupt change detection. In arXiv:1610.09626.

[48] Xiangyu Zhang, Xinyu Zhou, Mengxiao Lin, and Jian Sun. 2018. Shufflenet: An extremely efficient convolutional neural network for mobile devices. In Proceedings of the IEEE Conference on Computer Vision and Pattern Recognition. 6848-6856.

[49] D. Zhu, J. Choi, and R. W. Heath. 2017. Auxiliary beam pair enabled AoD and AoA estimation in closed-loop large-scale millimeter-wave MIMO system. In IEEE Transactions on Wireless Communications.

[50] Y. Zhu, S.J. Gortler, and D. Thurston. 2011. Sensor Network Localization Using Sensor Perturbation. ACM Transactions on Sensor Networks 7, 4, Article 36 (Feb. 2011), 23 pages. 